\title{
Millimeter-wave spectroscopy of chromium monochloride $(\mathrm{CrCl})$
}

\author{
Tomoko Oike, ${ }^{a}$ Toshiaki Okabayashi, and Mitsutoshi Tanimoto \\ Department of Chemistry, Faculty of Science, Shizuoka University, 836 Ohya, Shizuoka 422-8529, Japan
}

(Received 9 February 1998; accepted 27 May 1998)

\begin{abstract}
The rotational spectrum of chromium monochloride in the ${ }^{6} \Sigma^{+}$ground electronic state has been observed with a source-modulated microwave spectrometer in the millimeter-wave region. The $\mathrm{CrCl}$ radical was produced in a free space absorption cell by a dc glow discharge in a mixture of $\mathrm{AlCl}_{3}$ vapor and He using stainless steel electrodes. Chromium atom was supplied from the electrodes by the sputtering reaction. Observed spectral lines were analyzed by a least-squares fit and the molecular constants including the rotational and centrifugal distortion constants and the spin-spin and spin-rotation interaction constants were determined. The experimental rotational constants $B_{0}$ for ${ }^{52} \mathrm{Cr}^{35} \mathrm{Cl}$ and ${ }^{52} \mathrm{Cr}^{37} \mathrm{Cl}$ are 5009.345 69(69) and $4847.85100(32) \mathrm{MHz}$, respectively, where the uncertainties attached to the last digit correspond to one standard deviation. (c) 1998 American Institute of Physics. [S0021-9606(98)00933-7]
\end{abstract}

\section{INTRODUCTION}

High-resolution spectroscopy has so far been applied to the study on many species containing typical elements only. ${ }^{1,2}$ In these chemical species orbital degeneracy in the electronic ground state is never higher than 2 ( $\Pi$ or $E$ ), and spin multiplicity is usually lower than quartet. Since a transition metal atom contains a number of unpaired $d$ electrons, however, even for a simplest diatomic molecule the orbital degeneracy can be larger than 2 and the spin multiplicity higher than triplet. Such a species gives complex spectra and their analysis is accordingly difficult. Furthermore, since metal compounds, in general, are highly refractory, few methods are available to produce gaseous molecules efficiently for spectroscopic study at room temperature. These are some of the reasons why few species containing a transition metal atom have been studied with high-resolution spectroscopy.

Observation of the spectrum of such a molecule is useful not only to establish its existence in the gas phase and to obtain spectroscopic information. It also serves to check the accuracy of the molecular constants calculated with nonempirical molecular orbital methods and the reliability of the effective Hamiltonian used in the analysis.

A number of transition metal elements have relatively high cosmic abundance. Among hydrides of $\mathrm{Fe}, \mathrm{Cr}$, and the like, $\mathrm{CrH}$ was detected in relatively cool $S$-type stars and sunspots. ${ }^{3,4}$ Abundance of transition metal compounds (hydrides, oxides, halides, and so on) was calculated in a theoretical study based on chemical models of the reactions in stellar atmospheres. ${ }^{5}$ Such species, however, have rarely been observed in the interstellar and circumstellar spaces. Heavy metals are in general produced by nuclear reactions in the central region of a star. As a result of a supernova explosion or mass loss they are ejected toward the stellar atmosphere. Compounds including transition metal atoms could

\footnotetext{
${ }^{a)}$ Present address: Department of Astronomical Science, The Graduate University for Advanced Studies, c/o Nobeyama Radio Observatory, Minamimaki, Minamisaku, Nagano 384-13, Japan.
}

thus exist in the interstellar space, connected with interstellar dust. However, unless much detailed high-resolution spectroscopic data are accumulated, we cannot identify them. In this sense the study on rotational spectra of transition metal compounds is urgently desired.

Among the compounds bearing chromium, chromium hydride, $\mathrm{CrH},{ }^{6-11}$ and chromium monoxide, $\mathrm{CrO},{ }^{12-14}$ have extensively been investigated with various spectroscopic methods. Chromium monofluoride, $\mathrm{CrF}$, has also been the subject of recent studies through electronic spectroscopy of the $A^{6} \Sigma^{+}-X^{6} \Sigma^{+}$and $B{ }^{6} \Pi-X^{6} \Sigma^{+}$band systems with the resolved rotational structure ${ }^{15-17}$ and through microwave spectroscopy. ${ }^{18}$ On the other hand, for $\mathrm{CrCl}$ Ermilov et al. ${ }^{19}$ approached the energy levels of the electronic states theoretically and indicated that the molecule has the electronic ground $X^{6} \Sigma^{+}$state. Spectroscopically only low resolution emission spectra of electronic transitions were investigated. ${ }^{20}$ Quite recently Bencheikh et al. reported on the analysis of the $A-X$ electronic transition with high-resolution near infrared spectroscopy. ${ }^{21}$

Species containing a transition metal atom such as chromium have hardly been studied with microwave spectroscopy. ${ }^{1,2}$ In the present study we have observed the rotational spectrum of the high-spin molecular species, $\mathrm{CrCl}$, generated in a dc discharge plasma and have precisely determined its molecular constants, when no high-resolution works were available. ${ }^{22}$

\section{EXPERIMENT}

The measurement was carried out using a $100 \mathrm{kHz}$ source-modulated microwave spectrometer, which had been described in a previous paper. ${ }^{23}$ Millimeter-wave radiation was generated by a tripler driven by klystrons. A liquid $\mathrm{He}$ cooled $\mathrm{InSb}$ detector was employed to detect microwave power. A free space absorption cell was a $2 \mathrm{~m}$ long, $10 \mathrm{~cm}$ diameter Pyrex tube, which contained a pair of cylindrical stainless-steel plates as electrodes for discharge. The cell was wound by a copper wire, through which dc current flowed to compensate the Earth's magnetic field. 
The spectrum of the $\mathrm{CrCl}$ radical was incidentally observed ${ }^{22}$ while we were searching for rotational transition lines of $\mathrm{AlCl}$ in the $a^{3} \Pi$ first excited electronic state by dc glow discharging in a mixture of $\mathrm{AlCl}_{3}$ vapor and $\mathrm{He}$ gas. The experimental conditions on searching were $60 \mathrm{mTorr}$ of the total pressure and $600 \mathrm{~mA}$ of the discharge current which was optimized for the search of the $\mathrm{AlCl}$ lines. In the region of 267-272 GHz, we found 6 paramagnetic lines of similar intensity separated by about $50 \mathrm{MHz}$. Each line did not show up any further splittings due to magnetic and chlorine nuclear quadrupole hyperfine interactions. It seemed that the frequency separation was not appropriate for $\mathrm{AlCl}$ in the excited triplet electronic state, where the separation would be dependent on the spin sublevels. The carrier was guessed through the following chemical evidence. When we used $\mathrm{AlBr}_{3}$ instead of $\mathrm{AlCl}_{3}$, these lines could not be observed. On the other hand, use of chlorine-containing volatile molecules, such as $\mathrm{CCl}_{4}$ and $\mathrm{SiCl}_{4}$, yielded the same six line spectrum, whose intensity was weaker than that with $\mathrm{AlCl}_{3}$. This suggested that the six lines probably arose from a single species and that the plausibly new paramagnetic molecule contained a chlorine atom and an atom other than aluminum.

We then suspected that rather wide frequency separation resulted from fine structure in a high-spin species, which would be possible for a transition metal compound with several $d$ electrons. The transition metal atom could only be supplied into the system through sputtering of stainless steel discharge electrodes, sole metal components in the cell. Stainless steel has nominal composition of $\mathrm{Fe} 70 \%$, Cr 20\%, and $\mathrm{Ni} 10 \%$. So the metal atom having the ground or lowlying excited ${ }^{6} \Sigma$ state was supposed to be either $\mathrm{Cr}$ or $\mathrm{Fe}$. The plausible hyperfine interaction contribution decreases with increasing $J$ and the splittings are not so large for high $J$ transitions in the submillimeter-wave region. The $\Lambda$-doubling affects the pure rotational spectrum differently in each spin substate. Thus the observation of the rotational spectrum in various frequency regions yields a clue on the electronic state.

Experimental conditions were optimized for these lines: $P\left(\mathrm{AlCl}_{3}\right)=5 \mathrm{mTorr}, P(\mathrm{He})=50 \mathrm{mTorr}$, and discharge current of $700 \mathrm{~mA}$. The cell was kept at $50{ }^{\circ} \mathrm{C}$ to prevent $\mathrm{AlCl}_{3}$ from condensing on the cell wall. Higher temperature made the signal intensity stronger but caused the unstable discharge, apparently as gases adsorbed on the cell wall desorbed intermittently.

We observed a spectral pattern comprising equally intense 6 lines nearly every $10 \mathrm{GHz}$. Figure 1 shows a schematic pattern of the spectrum at eight different frequency regions. The frequency separations varied only slightly with rotational quantum numbers and were observed to be $30-80$ MHz. This experimental finding of equal intensity and separation suggested the ${ }^{6} \Sigma$ symmetry of the electronic state.

Inference of the electronic state gave no definite conclusion yet on what the molecular species was. To identify the molecule containing a chlorine atom we attempted to observe the rotational spectrum of a less abundant isotopic species (one-third of the parent species). For the two candidates of $\mathrm{CrCl}$ and $\mathrm{FeCl}$ we calculated the rotational constants and other spectroscopic constants of the chlorine isotopomer,

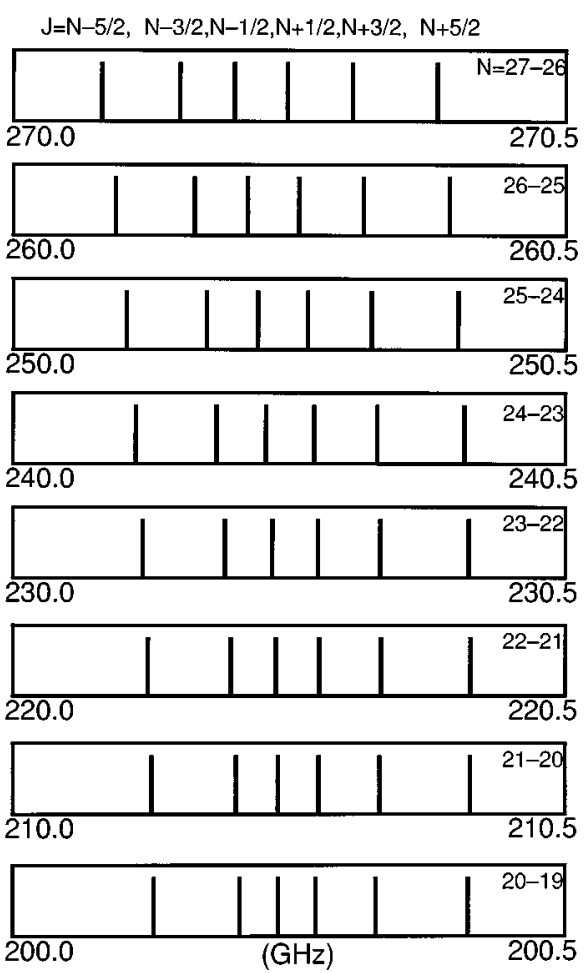

FIG. 1. A schematic spectral pattern of $\mathrm{CrCl}$ rotational transition shows that the separation between the component lines is nearly independent of rotational angular momentum. The splitting is thus due to fine structure interaction.

$\mathrm{Cr}^{37} \mathrm{Cl}$ and $\mathrm{Fe}{ }^{37} \mathrm{Cl}$, scaled from those of the ${ }^{35} \mathrm{Cl}$ species by the respective reduced masses. ${ }^{24} \mathrm{We}$ predicted the transition frequencies for the two molecules and were able to observe the $N=27-26, J=49 / 2-47 / 2$ transition in the region where the spectrum of the chromium species was expected to appear. Subsequent observation for other $J$ transitions was critical for the determination of the carrier as $\mathrm{CrCl}$ in the ${ }^{6} \Sigma^{+}$electronic ground state. This assignment of $\mathrm{CrCl}$ in this system $^{22}$ later stimulated the observation of the rotational spectrum of $\mathrm{FeCl}$ by Tanimoto et $\mathrm{al}^{25}$ and independently by Allen et $a l .{ }^{26}$

In the frequency region between 200 and $270 \mathrm{GHz}$, lines of the $\mathrm{Cr}^{35} \mathrm{Cl}$ radical were observed and assigned to rotational transitions with $N=20-19$ to $27-26$. Figure 2 illustrates a typical transition line of $\mathrm{Cr}^{35} \mathrm{Cl}$ near $230.2 \mathrm{GHz}$. It also shows that on application of a magnetic field of $50 \mathrm{G}$ the $\mathrm{CrCl}$ line disappears by Zeeman effect. The remaining line on the low frequency side is due to $\mathrm{Al}^{35} \mathrm{Cl}$ in a highly excited vibrational state of $v=11$, which lies at about $5600 \mathrm{~cm}^{-1}$ in energy. For the $\mathrm{Cr}^{37} \mathrm{Cl}$ species 21 lines were observed in the frequency region of 203 to $262 \mathrm{GHz}$. Tables I and II list the observed transition frequencies for the $\mathrm{Cr}^{35} \mathrm{Cl}$ and $\mathrm{Cr}^{37} \mathrm{Cl}$ species, respectively, along with their assignment.

\section{ANALYSIS}

The measured transition frequencies were analyzed by a least-squares method. Since the $\mathrm{CrCl}$ radical in its $X^{6} \Sigma^{+}$ ground state has five unpaired electrons, the analysis was 


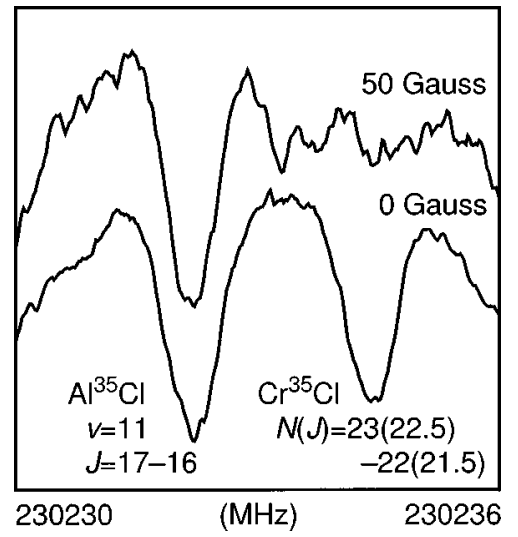

FIG. 2. A typical transition line of $\mathrm{Cr}^{35} \mathrm{Cl}$ near $230.2 \mathrm{GHz}$. On application of a magnetic field the $\mathrm{CrCl}$ line disappears by the Zeeman effect. The remaining line on the low frequency side is due to diamagnetic aluminum monochloride in a vibrationally excited state of $v=11$ in the electronic ground state.

carried out using the Hamiltonian of Hund's case (a) or (b) with the inclusion of the spin-rotation and spin-spin interactions:

$$
H=H_{R}+H_{\mathrm{SR}}+H_{\mathrm{SS}},
$$

where $H_{R}, H_{\mathrm{SR}}$, and $H_{\mathrm{SS}}$ are Hamiltonians for the rotation, spin-rotation, and spin-spin interactions, respectively. The explicit forms of the various terms are as follows:

$$
\begin{aligned}
& H_{R}=B \mathbf{N}^{2}-D \mathbf{N}^{4}, \\
& H_{\mathrm{SR}}=\gamma(\mathbf{N} \cdot \mathbf{S})+\gamma_{D}(\mathbf{N} \cdot \mathbf{S}) \mathbf{N}^{2}+\gamma_{H}(\mathbf{N} \cdot \mathbf{S}) \mathbf{N}^{4},
\end{aligned}
$$

and

$$
H_{\mathrm{SS}}=\frac{2}{3} \lambda\left(3 S_{z}-\mathbf{S}^{2}\right)+\frac{2}{3} \lambda_{D}\left(3 S_{z}-\mathbf{S}^{2}\right) \mathbf{N}^{2} .
$$

The matrix elements of the Hamiltonian in a case (a) basis set were given by Ram et al. ${ }^{11}$ and used in our case (a) treatment. The higher-order term in the spin-orbit interaction $H_{\text {SO }}^{(4)}$ was also included in the analysis: ${ }^{9}$

$$
H_{\mathrm{SO}}^{(4)}=\frac{1}{12} \theta\left[35 S_{z}^{2}-30 \mathbf{S}^{2} S_{z}^{2}+25 S_{z}^{2}-6 \mathbf{S}^{2}+3 \mathbf{S}^{4}\right] .
$$

The molecular constants determined are given in Table III. Calculated frequencies are listed in Tables I and II. The standard deviations of the fit are 39 and $41 \mathrm{kHz}$ for $\mathrm{Cr}^{35} \mathrm{Cl}$ and $\mathrm{Cr}^{37} \mathrm{Cl}$, respectively.

On the other hand, the more appropriate basis set for molecules in a $\Sigma$ state will be a Hund's case (b) basis set. In a $\Sigma$ state, electron spins do not couple to the molecular axis, but to the axis of rotation through a weak magnetic field generated by the end-over-end rotation of the molecule. We also calculated the matrix elements in a case (b) representation. The matrix elements of the spin-rotation interaction for $\mathrm{a}^{6} \Sigma$ state in this basis are

$$
\begin{aligned}
\left\langle N^{\prime} S J^{\prime}\left|H_{\mathrm{SR}}\right| N S J\right\rangle= & \frac{1}{2}\left[\gamma+\gamma_{D} N(N+1)\right] \\
& \times[J(J+1)-N(N+1) \\
& -S(S+1)] \delta_{N^{\prime} N} \delta_{J^{\prime} J},
\end{aligned}
$$

where $\delta_{N^{\prime} N}$ is Kronecker's delta and those of the spin-spin interaction are

$$
\begin{aligned}
\left\langle N^{\prime} S J^{\prime}\left|H_{\mathrm{SS}}\right| N S J\right\rangle= & -\frac{2 \sqrt{30}}{2} \lambda_{\text {eff }}(-1)^{S+N+J}\left\{\begin{array}{lll}
J & N & S \\
2 & S & N
\end{array}\right\}\left\{\begin{array}{lll}
1 & 1 & 2 \\
S & S & S
\end{array}\right\} S(S+1)(2 S+1) \sqrt{\frac{N(N+1)(2 N+1)}{(2 N-1)(2 N+3)}} \delta_{N^{\prime}, N} \\
& -2 \sqrt{5} \lambda_{\text {eff }}(-1)^{S+N+J}\left\{\begin{array}{ccc}
J & N+2 & S \\
2 & S & N
\end{array}\right\}\left\{\begin{array}{lll}
1 & 1 & 2 \\
S & S & S
\end{array}\right\} S(S+1)(2 S+1) \sqrt{\frac{(N+1)(2 N+1)}{(2 N+3)}} \delta_{N^{\prime}, N+2} \\
& -2 \sqrt{5} \lambda_{\text {eff }}(-1)^{S+N+J}\left\{\begin{array}{ccc}
J & N-2 & S \\
2 & S & N
\end{array}\right\}\left\{\begin{array}{lll}
1 & 1 & 2 \\
S & S & S
\end{array}\right\} S(S+1)(2 S+1) \sqrt{\frac{N(N-1)}{(2 N-1)}} \delta_{N^{\prime}, N-2} .
\end{aligned}
$$

The term $H_{\mathrm{SS}}$ possesses off-diagonal elements with respect to $S_{z}$. The term in the curly brackets in this expression represents a 6- $j$ symbol. The coefficient $\lambda_{\text {eff }}$ is not a constant in the individual matrix elements but includes a term dependent on $N$, since the molecule suffers from the perturbation due to the centrifugal distortion. This was treated by Mizushima and Hill ${ }^{27}$ for the analysis of the $\mathrm{O}_{2}$ microwave spectrum. The general expression for $\lambda_{\text {eff }}$ is as follows:

$$
\lambda_{\mathrm{eff}}=\lambda+\lambda_{D} \mathbf{N}^{2}
$$

The matrix elements of the spin-spin coupling constant $\lambda_{\text {eff }}$ are thus seen to be

$$
\lambda_{\mathrm{eff}} \equiv \lambda+N(N+1) \lambda_{D}
$$

for the $\langle N \mid N\rangle$ elements, and

$$
\lambda_{\text {eff }} \equiv \lambda+\left(N^{2}+N+1\right) \lambda_{D}
$$

for the $\langle N-1 \mid N+1\rangle$ elements.

The matrix elements of the spin-orbit interaction $H_{\mathrm{SO}}^{(4)}$ were given by the equation: 
TABLE I. Observed transitions of ${ }^{52} \mathrm{Cr}^{35} \mathrm{Cl}$ in case (a) and (b).

\begin{tabular}{|c|c|c|c|c|c|c|c|c|c|}
\hline$N^{\prime}$ & $J^{\prime}$ & - & $N^{\prime \prime}$ & $J^{\prime \prime}$ & $\begin{array}{l}\text { Obs. Freq. } \\
/ \mathrm{MHz}\end{array}$ & $\begin{array}{c}\text { Calc. Freq.(a) } \\
/ \mathrm{MHz}\end{array}$ & $\begin{array}{c}\mathrm{o}-\mathrm{c}^{\mathrm{a}}(\mathrm{a}) \\
/ \mathrm{kHz}\end{array}$ & $\begin{array}{c}\text { Calc. Freq.(b) } \\
/ \mathrm{MHz}\end{array}$ & $\begin{array}{c}o-c^{a}(b) \\
/ k H z\end{array}$ \\
\hline 20 & $35 / 2$ & & 19 & $33 / 2$ & 200127.714 & 200127.773 & -59 & 200127.735 & -21 \\
\hline 20 & $37 / 2$ & & 19 & $35 / 2$ & 200205.627 & 200205.617 & 10 & 200205.666 & -39 \\
\hline 20 & $39 / 2$ & & 19 & $37 / 2$ & 200240.420 & 200240.442 & -22 & 200240.451 & -31 \\
\hline 20 & $41 / 2$ & & 19 & $39 / 2$ & 200274.359 & 200274.423 & -64 & 200274.389 & -30 \\
\hline 20 & $43 / 2$ & & 19 & $41 / 2$ & 200328.678 & 200328.697 & -19 & 200328.683 & -5 \\
\hline 20 & $45 / 2$ & & 19 & $43 / 2$ & 200411.925 & 200411.909 & 16 & 200411.947 & -22 \\
\hline 21 & $37 / 2$ & & 20 & $35 / 2$ & 210125.605 & 210125.619 & -14 & 210125.589 & 16 \\
\hline 21 & $39 / 2$ & & 20 & $37 / 2$ & 210202.170 & 210202.127 & 43 & 210202.158 & 12 \\
\hline 21 & $41 / 2$ & & 20 & $39 / 2$ & 210240.094 & 210240.123 & -29 & 210240.122 & -28 \\
\hline 21 & $43 / 2$ & & 20 & $41 / 2$ & 210276.969 & 210276.968 & 1 & 210276.947 & 22 \\
\hline 21 & $45 / 2$ & & 20 & $43 / 2$ & 210332.150 & 210332.126 & 24 & 210332.123 & 27 \\
\hline 21 & $47 / 2$ & & 20 & $45 / 2$ & 210414.047 & 210414.015 & 32 & 210414.039 & 8 \\
\hline 22 & $39 / 2$ & & 21 & $37 / 2$ & 220122.150 & 220122.186 & -36 & 220122.166 & -16 \\
\hline 22 & $41 / 2$ & & 21 & $39 / 2$ & 220197.496 & 220197.498 & -2 & 220197.516 & -20 \\
\hline 22 & $43 / 2$ & & 21 & $41 / 2$ & 220238.171 & 220238.166 & 5 & 220238.155 & 16 \\
\hline 22 & $45 / 2$ & & 21 & $43 / 2$ & 220277.481 & 220277.493 & -12 & 220277.483 & -2 \\
\hline 22 & $47 / 2$ & & 21 & $45 / 2$ & 220333.426 & 220333.455 & -29 & 220333.461 & -35 \\
\hline 22 & $49 / 2$ & & 21 & $47 / 2$ & 220414.201 & 220414.206 & -5 & 220414.217 & -16 \\
\hline 23 & $41 / 2$ & & 22 & $39 / 2$ & 230117.280 & 230117.289 & -9 & 230117.283 & -3 \\
\hline 23 & $43 / 2$ & & 22 & $41 / 2$ & 230191.606 & 230191.530 & 76 & 230191.537 & 69 \\
\hline 23 & $45 / 2$ & & 22 & $43 / 2$ & 230234.510 & 230234.465 & 45 & 230234.446 & 64 \\
\hline 23 & $47 / 2$ & & 22 & $45 / 2$ & 230275.954 & 230275.956 & -2 & 230275.957 & -3 \\
\hline 23 & $49 / 2$ & & 22 & $47 / 2$ & 230332.657 & 230332.651 & 6 & 230332.665 & -8 \\
\hline 23 & $51 / 2$ & & 22 & $49 / 2$ & 230412.466 & 230412.420 & 46 & 230412.416 & 50 \\
\hline 24 & $43 / 2$ & & 23 & $41 / 2$ & 240110.816 & 240110.769 & 47 & 240110.777 & 39 \\
\hline 24 & $45 / 2$ & & 23 & $43 / 2$ & 240184.025 & 240184.043 & -18 & 240184.042 & -17 \\
\hline 24 & $47 / 2$ & & 23 & $45 / 2$ & 240228.912 & 240228.916 & -4 & 240228.890 & 22 \\
\hline 24 & $49 / 2$ & & 23 & $47 / 2$ & 240272.355 & 240272.305 & 50 & 240272.317 & 38 \\
\hline 24 & $51 / 2$ & & 23 & $49 / 2$ & 240329.683 & 240329.670 & 13 & 240329.692 & -9 \\
\hline 24 & $53 / 2$ & & 23 & $51 / 2$ & 240408.553 & 240408.586 & -33 & 240408.567 & -14 \\
\hline 25 & $45 / 2$ & & 24 & $43 / 2$ & 250102.523 & 250102.482 & 41 & 250102.507 & 16 \\
\hline 25 & $47 / 2$ & & 24 & $45 / 2$ & 250174.899 & 250174.881 & 18 & 250174.874 & 25 \\
\hline 25 & $49 / 2$ & & 24 & $47 / 2$ & 250221.402 & 250221.422 & -20 & 250221.390 & 12 \\
\hline 25 & $51 / 2$ & & 24 & $49 / 2$ & 250266.552 & 250266.483 & 69 & 250266.505 & 47 \\
\hline 25 & $53 / 2$ & & 24 & $51 / 2$ & 250324.483 & 250324.461 & 22 & 250324.490 & -7 \\
\hline 25 & $55 / 2$ & & 24 & $53 / 2$ & 250402.618 & 250402.635 & -17 & 250402.599 & 19 \\
\hline 26 & $47 / 2$ & & 25 & $45 / 2$ & 260092.320 & 260092.297 & 23 & 260092.342 & -22 \\
\hline 26 & $49 / 2$ & & 25 & $47 / 2$ & 260163.819 & 260163.901 & -82 & 260163.890 & -71 \\
\hline 26 & $51 / 2$ & & 25 & $49 / 2$ & 260211.829 & 260211.886 & -57 & 260211.848 & -19 \\
\hline 26 & $53 / 2$ & & 25 & $51 / 2$ & 260258.425 & 260258.426 & -1 & 260258.459 & -34 \\
\hline 26 & $55 / 2$ & & 25 & $53 / 2$ & 260316.963 & 260316.967 & -4 & 260317.001 & -38 \\
\hline 26 & $57 / 2$ & & 25 & $55 / 2$ & 260394.441 & 260394.493 & -52 & 260394.439 & 2 \\
\hline 27 & $49 / 2$ & & 26 & $47 / 2$ & 270079.954 & 270080.095 & $-141^{\mathrm{b}}$ & 270080.159 & $-205^{\mathrm{b}}$ \\
\hline 27 & $51 / 2$ & & 26 & $49 / 2$ & 270150.719 & 270150.972 & $-253^{b}$ & 270150.958 & $-239^{\mathrm{b}}$ \\
\hline 27 & $53 / 2$ & & 26 & $51 / 2$ & 270200.110 & 270200.213 & $-103^{b}$ & 270200.169 & $-59^{\mathrm{b}}$ \\
\hline 27 & $55 / 2$ & & 26 & $53 / 2$ & 270247.931 & 270248.068 & $-137^{b}$ & 270248.110 & $-179^{b}$ \\
\hline 27 & $57 / 2$ & & 26 & $55 / 2$ & 270307.047 & 270307.126 & $-79^{\mathrm{b}}$ & 270307.166 & $-119^{b}$ \\
\hline 27 & $59 / 2$ & & 26 & $57 / 2$ & 270383.795 & 270384.083 & $-288^{\mathrm{b}}$ & 270384.011 & $-216^{\mathrm{b}}$ \\
\hline
\end{tabular}

${ }^{a}$ Observed minus calculated transition frequencies. b

${ }^{\mathrm{b}}$ Rough measurement. Not included in the least-squares analysis.

$$
\begin{aligned}
\left\langle N^{\prime} \Lambda^{\prime} S J^{\prime}\left|H_{\mathrm{SO}}^{(4)}\right| N \Lambda S J\right\rangle= & \frac{\theta}{24}(-1)^{N+S+J}\left\{\begin{array}{ccc}
J & S & N^{\prime} \\
4 & N & S
\end{array}\right\}(-1)^{N^{\prime}-\Lambda^{\prime}}\left(\begin{array}{ccc}
N^{\prime} & 4 & N \\
-\Lambda & 0 & \Lambda
\end{array}\right) \sqrt{\left(2 N^{\prime}+1\right)(2 N+1)} \\
& \times \sqrt{(2 S-3)(2 S-2)(2 S-1) 2 S(2 S+1)(2 S+2)(2 S+3)(2 S+4)(2 S+5)} \delta_{J^{\prime} J} .
\end{aligned}
$$


TABLE II. Observed transitions of ${ }^{52} \mathrm{Cr}^{37} \mathrm{Cl}$.

\begin{tabular}{|c|c|c|c|c|c|c|c|c|c|}
\hline$N^{\prime}$ & $J^{\prime}$ & - & $N^{\prime \prime}$ & $J^{\prime \prime}$ & $\begin{array}{l}\text { Obs. Freq. } \\
\text { /MHz }\end{array}$ & $\begin{array}{c}\text { Calc. Freq.(a) } \\
\text { /MHz }\end{array}$ & $\begin{array}{c}o-c^{a}(a) \\
/ k H z\end{array}$ & $\begin{array}{c}\text { Calc. Freq.(b) } \\
/ \mathrm{MHz}\end{array}$ & $\begin{array}{c}\mathrm{o}-\mathrm{c}^{\mathrm{a}}(\mathrm{b}) \\
/ \mathrm{kHz}\end{array}$ \\
\hline 21 & $43 / 2$ & & 20 & $41 / 2$ & 203503.423 & 203503.457 & -34 & 203503.425 & -2 \\
\hline 21 & $45 / 2$ & & 20 & $43 / 2$ & 203558.246 & 203558.271 & -25 & 203558.257 & -11 \\
\hline 21 & $47 / 2$ & & 20 & $45 / 2$ & 203636.106 & 203636.081 & 25 & 203636.116 & -10 \\
\hline 22 & $39 / 2$ & & 21 & $37 / 2$ & 213028.106 & 213028.131 & -25 & 213028.098 & 8 \\
\hline 22 & $41 / 2$ & & 21 & $39 / 2$ & 213099.728 & 213099.693 & 35 & 213099.735 & -7 \\
\hline 22 & $43 / 2$ & & 21 & $41 / 2$ & 213141.251 & 213141.272 & -21 & 213141.266 & -15 \\
\hline 22 & $45 / 2$ & & 21 & $43 / 2$ & 213182.050 & 213182.044 & 6 & 213182.029 & 21 \\
\hline 22 & $47 / 2$ & & 21 & $45 / 2$ & 213237.523 & 213237.522 & 1 & 213237.523 & 0 \\
\hline 22 & $49 / 2$ & & 21 & $47 / 2$ & 213314.376 & 213314.359 & 17 & 213314.378 & -2 \\
\hline 23 & $41 / 2$ & & 22 & $39 / 2$ & 222701.856 & 222701.892 & -36 & 222701.871 & -15 \\
\hline 23 & $43 / 2$ & & 22 & $41 / 2$ & 222772.565 & 222772.530 & 35 & 222772.555 & 10 \\
\hline 23 & $45 / 2$ & & 22 & $43 / 2$ & 222816.084 & 222816.088 & 4 & 222816.071 & 13 \\
\hline 23 & $47 / 2$ & & 22 & $45 / 2$ & 222858.705 & 222858.711 & -6 & 222858.711 & -6 \\
\hline 23 & $49 / 2$ & & 22 & $47 / 2$ & 222914.825 & 222914.797 & 28 & 222914.809 & 16 \\
\hline 23 & $51 / 2$ & & 22 & $49 / 2$ & 222990.804 & 222990.793 & 11 & 222990.795 & 9 \\
\hline 27 & $49 / 2$ & & 26 & $47 / 2$ & 261380.333 & 261380.280 & 53 & 261380.329 & 4 \\
\hline 27 & $51 / 2$ & & 26 & $49 / 2$ & 261447.980 & 261447.999 & -19 & 261447.980 & 0 \\
\hline 27 & $53 / 2$ & & 26 & $51 / 2$ & 261496.991 & 261497.038 & -47 & 261496.985 & 6 \\
\hline 27 & $55 / 2$ & & 26 & $53 / 2$ & 261545.128 & 261545.094 & 34 & 261545.146 & -18 \\
\hline 27 & $57 / 2$ & & 26 & $55 / 2$ & 261603.191 & 261603.145 & 46 & 261603.193 & -2 \\
\hline 27 & $59 / 2$ & & 26 & $57 / 2$ & 261676.669 & 261676.745 & -76 & 261676.666 & 3 \\
\hline
\end{tabular}

${ }^{\mathrm{a}}$ Observed minus calculated transition frequencies.

We calculated energy levels by diagonalizing the Hamiltonian matrix and determined the molecular constants by a least-squares fit to the observed transition frequencies. The calculated frequencies from the case (b) analysis are included in Tables I and II. The standard deviations of the fit are 32 and $13 \mathrm{kHz}$ for $\mathrm{Cr}^{35} \mathrm{Cl}$ and $\mathrm{Cr}^{37} \mathrm{Cl}$, respectively. The molecular constants in the case (b) are given in the fourth and fifth columns of Table III.

\section{RESULTS AND DISCUSSION}

We have observed the rotational spectrum for both chlorine isotopic species of the high-spin radical $\mathrm{CrCl}$ and determined its precise molecular constants. The ground-state rotational constant $B_{0}$ agrees with the near-infrared value ${ }^{21}$ within their $3 \sigma$, when their $B_{e}$ value is converted into $B_{0}$ using the vibration-rotation interaction constant $\alpha$. The rotational constants $B_{0}$ give the interatomic distances of $r_{0}$ $=2.1971403$ (9) $\AA$ and 2.1970846 (9) $\AA$ for $\mathrm{Cr}^{35} \mathrm{Cl}$ and $\mathrm{Cr}^{37} \mathrm{Cl}$, respectively. This bond length is slightly shorter than the $\mathrm{Cr}-\mathrm{Cl}$ distance of monomeric chromium dichloride (2.207 (10) $\AA$ ) determined by Hargittai et al. with electron diffraction in vapor phase. ${ }^{28}$ The $\mathrm{Cr}-\mathrm{Cl}$ distance for $\mathrm{CrCl}_{2}$ in a nitrogen matrix, on the other hand, was determined with x-ray absorption fine structure (XAFS) spectroscopy to be 2.257 (2) $\AA{ }^{29}$ substantially longer than the gas phase value. The corresponding interatomic distance in the crystalline compound $\mathrm{CrCl}_{3}$ is $2.340-2.347 \AA$ for the monoclinic crystal structure at room temperature. ${ }^{30}$ It seems that addition of a chlorine atom to the chromium atom causes the elongation of the metal-halide bond length. The $r_{0}$ bond length of $\mathrm{CrCl}$ is about $0.02 \AA$ longer than that of $\mathrm{FeCl}\left(r_{0}\right.$ $=2.176987(1) \AA)$.

When the present ground state rotational constant is combined with the vibration-rotation interaction constant $\alpha$ (29.13 MHz) reported by Bencheikh et al. ${ }^{21}$ the equilibrium interatomic distance is determined to be 2.193952 (2) $\AA$.

The equilibrium bond length can also be estimated from the isotopically invariant Dunham constant $U_{01}$. This constant is estimated from the rotational constants for two isotopic species under the assumption that the anharmonicity in the potential function contributes negligibly to the lower order Dunham constants. This procedure yields the equilibrium bond length of 2.19378 (1) $\AA$. This distance is about $0.00018 \AA$ shorter than the value derived above. Thus the

TABLE III. Molecular constants of the $\mathrm{CrCl}$ radical.

\begin{tabular}{lcccc}
\hline \hline & \multicolumn{2}{c}{ Hund's Case (a) } & \multicolumn{2}{c}{ Hund's Case (b) } \\
& ${ }^{52} \mathrm{Cr}^{35} \mathrm{Cl}$ & ${ }^{52} \mathrm{Cr}^{37} \mathrm{Cl}$ & ${ }^{52} \mathrm{Cr}^{35} \mathrm{Cl}$ & ${ }^{52} \mathrm{Cr}^{37} \mathrm{Cl}$ \\
\hline$B_{0} / \mathrm{MHz}$ & $5009.34695(81)$ & $4847.8524(10)$ & $5009.34569(69)$ & $4847.85100(32)$ \\
$D_{0} / \mathrm{kHz}$ & $3.52892(72)$ & $3.30613(83)$ & $3.52846(60)$ & $3.30573(26)$ \\
$\gamma / \mathrm{MHz}$ & $65.534(22)$ & $63.462(31)$ & $65.646(26)$ & $63.607(13)$ \\
$\gamma_{D} / \mathrm{kHz}$ & $-0.0839(87)$ & $-0.105(11)$ & $-0.151(12)$ & $-0.1849(57)$ \\
$\lambda / \mathrm{MHz}$ & $7992.5(27)$ & $7997.1(39)$ & $7981.0(25)$ & $7981.5(14)$ \\
$\lambda_{D} / \mathrm{kHz}$ & $-13.214(54)$ & $-12.752(78)$ & $-13.249(45)$ & $-12.746(24)$ \\
$\theta / \mathrm{MHz}$ & $-2.11(82)$ & $-2.7(12)$ & $-3.24(63)$ & $-3.86(34)$ \\
\hline \hline
\end{tabular}


effect of anharmonicity on the equilibrium distance was of the order of $0.0002 \AA$ in this molecule. This procedure can be used to estimate approximate $r_{e}$ from the data for two isotopomers, when the constant $\alpha$ is not available.

The fundamental vibrational frequency $\omega_{e}$ is related to the equilibrium rotational constant $B_{e}$ and the centrifugal distortion constant $D_{e}$ through the relation ${ }^{24} \omega_{e}=2 \sqrt{B_{e}^{3} / D_{e}}$. This relation is approximately valid, even when the equilibrium constants are replaced by the effective constants in the ground state; thus the vibrational frequencies $\omega_{e}$ for $\mathrm{Cr}^{35} \mathrm{Cl}$ and $\mathrm{Cr}^{37} \mathrm{Cl}$ are estimated to be 398.2 and $391.7 \mathrm{~cm}^{-1}$, respectively. The former is favorably compared with the frequency $396.662 \mathrm{~cm}^{-1}$ from the electronic spectroscopy. ${ }^{21}$

The spin-rotation interaction constant $\gamma$ determined in the present study is considerably smaller than that for the $\mathrm{CrF}$ radical. The ratio $\gamma / B$ for the $\mathrm{CrCl}$ radical is about $40 \%$ of that for $\mathrm{CrF}$. The spin-rotation interaction arises from the microscopic electron spin-nuclear rotation interaction $\gamma^{(1)}$ and from second-order interactions with other electronic states $\gamma^{(2)} \cdot{ }^{31,32}$ In heavy molecules the second-order contribution is dominant. A few electronic states have been assessed experimentally by electronic spectroscopy and theoretically by density functional theory (DFT) and ligand field theory (LFT) treatments. ${ }^{21}$ The electronic energy level scheme of $\mathrm{CrCl}$ and $\mathrm{CrF}$ resembles each other and the lowest excited state is in both molecules the $B{ }^{6} \Pi$ state, which lies slightly below the $A^{6} \Sigma^{+}$state, the upper state of the electronic transition analyzed experimentally. If the single perturber approximation is valid and the interacting state is the lowest ${ }^{6} \Pi$ state, the difference in $\gamma / B$ for both molecules amounts to about $10 \%$. This suggests that the $\gamma$ constant results from the interaction with various higher energy states in addition to the experimentally known lower states.

The observed spin-spin interaction constant of $\mathrm{CrCl}$ (7981.0 MHz) is about half the value for $\mathrm{CrF}$ (16 162.4 $\mathrm{MHz}){ }^{18}$ The spin-spin interaction also results from a direct first-order interaction and second-order spin-orbit interactions. The second-order contribution from the spin-orbit interaction will be dominant in heavy molecules like $\mathrm{CrCl}$. Experimentally or theoretically assessed electronic excited ${ }^{4} \Sigma^{+}$states ${ }^{19,21,33}$ do not interact with the ground state by the spin-orbit interaction because of the \pm reflection symmetry selection rule. The interaction with the nearest $B^{6} \Pi$ state cannot explain the difference in $\lambda$ for both molecules, since the transition energies to this excited state from the ground state do not differ significantly. The experimentally unknown ${ }^{4} \Sigma^{-}$state may be important for this interaction.

All observed lines are single with no magnetic and nuclear quadrupole hyperfine splitting observed. Since the $\mathrm{CrCl}$ species has a highly ionic character, represented as $\mathrm{Cr}^{+} \mathrm{Cl}^{-}$, as exemplified by the molecular orbital calculation, ${ }^{21}$ the five unpaired electrons probably reside mainly near the chromium nucleus and one electron is transferred to the chlorine atom. Consequently the chlorine nucleus was approximately surrounded by the spherical distributions of the electrons and hence the quadrupole hyperfine interaction becomes small. Magnetic hyperfine interaction depends on the distance between the unpaired electron and the nucleus with a nuclear spin. In the ionic structure suggested above the relevant distance is rather large and also the magnetic hyperfine structure is small. This is consistent with the appearance of the spectral lines as a single line in the observed frequency regions.

In the present study the least-squares analysis was made in two ways, in terms of the case (a) and (b) basis sets. Initial analysis was made using the wave functions in Hund's case (a). This analysis showed that addition of observed transitions in the higher frequency region into the analysis affected the lower-order molecular constants as well as higher order centrifugal distortion corrections, although the residuals remained in the same order of magnitude. We thus calculated the rotational energy in the case (b) basis. In this case the anomalous behavior in the least-squares analysis was not detected. The resulting residuals were slightly smaller as compared with the case (a) basis calculations when the same order of the centrifugal corrections were included.

As described in the Experiment we used $\mathrm{AlCl}_{3}$ to observe the rotational spectrum of $\mathrm{CrCl}$. The cell temperature was maintained at about $50{ }^{\circ} \mathrm{C}$. Replacement of $\mathrm{AlCl}_{3}$ with more volatile compounds required the cell cooled to temperatures below the melting point of the compounds. This may mean that some precursor molecules formed on the surface are involved in the formation of transition metal compounds.

\section{ACKNOWLEDGMENT}

The present study was supported by a Grant-in-Aid from the Ministry of Education, Science, Sports and Culture (No. 07217209, 07454150, 07740456 and 08740457).

${ }^{1}$ E. Hirota, Chem. Rev. 92, 141 (1992).

${ }^{2}$ E. Hirota, Annu. Rep. Prog. Chem., Sect. C: Phys. Chem. 91, 3 (1994).

${ }^{3}$ B. Lindgren and G. Olofsson, Astron. Astrophys. 84, 300 (1980).

${ }^{4}$ O. Engvold, H. Wöhl, and J. W. Brault, Astron. Astrophys., Suppl. Ser. 42, 209 (1980).

${ }^{5}$ T. Tsuji, Astron. Astrophys. 23, 411 (1973).

${ }^{6}$ B. Kleman and B. Liljeqvist, Ark. Fys. 9, 345 (1954).

${ }^{7}$ B. Kleman and U. Uhler, Can. J. Phys. 37, 537 (1959).

${ }^{8}$ S. O'Connor, Proc. R. Ir. Acad. Sect. A, Math. Astron. Phys. Sci. 65, 95 (1967)

${ }^{9}$ S. M. Corkery, J. M. Brown, S. P. Beaton, and K. M. Evenson, J. Mol. Spectrosc. 149, 257 (1991)

${ }^{10}$ K. Lipus, E. Bauchem, and W. Urban, Mol. Phys. 73, 1041 (1991).

${ }^{11}$ R. S. Ram, C. N. Jarman, and P. F. Bernath, J. Mol. Spectrosc. 161, 445 (1993).

${ }^{12}$ W. H. Hocking, A. J. Merer, D. J. Milton, W. E. Jones, and G. Krishnamurty, Can. J. Phys. 58, 516 (1980).

${ }^{13}$ A. S.-C. Cheung, W. Zyrnicki, and A. J. Merer, J. Mol. Spectrosc. 104, 315 (1984).

${ }^{14}$ S. P. Bagare and N. S. Murthy, Physica B \& C 125, 265 (1984).

${ }^{15}$ V. M. Dubov, D. V. Tschechovskoy, E. A. Shenyavskaya, and I. Kovács, Acta Phys. Hung. 65, 411 (1989).

${ }^{16}$ O. Launila, J. Mol. Spectrosc. 169, 373 (1995).

${ }^{17}$ S. Wallin, R. Koivisto, and O. Launila, J. Chem. Phys. 105, 388 (1996).

${ }^{18}$ T. Okabayashi and M. Tanimoto, J. Chem. Phys. 105, 7421 (1996).

${ }^{19}$ A. Y. Ermilov, A. V. Nemukhin, and N. F. Stepanov, Zh. Fiz. Khim. 65, 707 (1991).

${ }^{20}$ V. R. Rao and K. R. Rao, Indian J. Phys. 23, 508 (1949).

${ }^{21}$ M. Bencheikh, R. Koivisto, O. Launila, and J. P. Flament, J. Chem. Phys. 106, 6231 (1997).

${ }^{22}$ T. Oike, T. Okabayashi, and M. Tanimoto, Astrophys. J. 445, L67 (1995).

${ }^{23}$ T. Okabayashi and M. Tanimoto, J. Chem. Phys. 99, 3268 (1993).

${ }^{24}$ W. Gordy and R. L. Cook, Microwave Molecular Spectra, 3rd ed. (Wiley, New York, 1984). 
${ }^{25}$ M. Tanimoto, S. Saito, and T. Okabayashi, Chem. Phys. Lett. 242, 153 (1995).

${ }^{26}$ M. D. Allen, B. Z. Li, and L. M. Ziurys, Chem. Phys. Lett. 270, 517 (1997).

${ }^{27}$ M. Mizushima and R. M. Hill, Phys. Rev. 93, 745 (1954).

${ }^{28}$ M. Hargittai, O. V. Dorofeeva, and J. Trommel, Inorg. Chem. 24, 3963 (1993).
${ }^{29}$ I. R. Beattie, M. D. Spicer, and N. A. Young, J. Chem. Phys. 100, 8700 (1994).

${ }^{30}$ B. Morosin and A. Narath, J. Chem. Phys. 40, 1958 (1964).

${ }^{31}$ T. D. Varberg, J. A. Gray, R. W. Field, and A. J. Merer, J. Mol. Spectrosc. 156, 296 (1992).

${ }^{32}$ T. Nelis, J. M. Brown, and K. M. Evenson, J. Chem. Phys. 92, 4067 (1990).

${ }^{33}$ T. C. Devore, M. McQuaid, and J. L. Gole, High. Temp. Sci. 29, 1 (1990). 